\title{
Dental management of patients with inherited bleeding disorders: a retrospective study
}

SADJ September 2018, Vol. 73 No. 8 p500 - p506

SP Mahlangu', J Mahlangu², SL Shangase ${ }^{3}$

\section{ABSTRACT}

Introduction

Minor invasive procedures can precipitate a prolonged bleeding episode in patients who have inherited bleeding disorders (IBD). Protocols have been established to minimize this postoperative bleeding, and the management of dental procedures performed on patients with IBD have been reviewed internationally. This paper undertakes a review of the topic and is probably the first of this nature in South Africa.

\section{Aim and study design}

This retrospective study reviewed dental procedures performed over a 16-year period on patients with IBD at the Charlotte Maxeke Johannesburg Academic Hospital (CMJAH), and compared those treatment approaches with published international guidelines.

\section{Methods}

The data were extracted from the files of patients seen at the Haemophilia Comprehensive Care Centre (HCCC) and from the Wits Oral Health Centre (WOHC) database at $\mathrm{CMJAH}$.

\section{Results}

Dental extractions accounted for $58 \%$ of dental procedures performed.Local haemostatic agents were used in $42 \%$ of the study population.

\section{Author affiliations:}

1. Sibongile P. Mahlangu, BChD (University of the Western Cape), MSc (Dent) (University of the Witwatersrand), Dentist/Lecturer, Department of Oral Medicine and Periodontology, School of Oral Health Sciences, University of the Witwatersrand, Johannesburg.

2. Johnny Mahlangu, BSc, MBBCh, MMed, FCPath, Cert Clin Haem Head of School: School of Pathology, Director of Bleeding Disorders Unit: Charlotte Maxeke Johannesburg Academic Hospital, University of the Witwatersrand, Johannesburg.

3. Sindisiwe L. Shangase, MDent (Oral Medicine and Periodontics) (Medunsa), Head of Department: Oral Medicine and Periodontology, School of Oral Health Sciences, University of the Witwatersrand, Johannesburg.

Tel: +27 (0)11488 4887/ +27 (0)723952335

Email address: Sindisiwe.Shangase@wits.ac.za

Corresponding author: Sindisiwe L. Shangase

Head of Department: Oral Medicine and Perio-dontology, School of Oral

Health Sciences, University of the Witwatersrand, Johannesburg.

Email address: Sindisiwe.Shangase@wits.ac.za

$\begin{array}{ll}\text { ACRONYMS } \\ \text { CMJAH: } & \text { Charlotte Maxeke Johannesburg } \\ & \text { Academic Hospital } \\ \text { DDAVP: } & \text { Desmopressin } \\ \text { FRT: } & \text { Factor Replacement Therapy } \\ \text { GA: } & \text { General Anaesthesia } \\ \text { HA: } & \text { Haemophilia A } \\ \text { HCCC: } & \text { Haemophilia Comprehensive Care Centre } \\ \text { IBD: } & \text { Inherited Bleeding Disorders } \\ \text { LA: } & \text { Local Anaesthesia } \\ \text { TA: } & \text { Tranexamic Acid } \\ \text { VWD: } & \text { Von Willebrand Disease } \\ \text { WOHC: } & \text { Wits Oral Health Centre }\end{array}$

Postoperative bleeding was observed in three subjects, and the management of these correlated with the published guidelines.

\section{Conclusion}

In this single centre review, bleeding following dental procedures was infrequent. The recommended improvements include greater use of preventive and supportive dental procedures; and administration of local haemostatic agents in invasive procedures.

\section{Keywords}

Inherited bleeding disorders; Haemophilia; Von Willebrand Disease

\section{INTRODUCTION}

Patients with inherited bleeding disorders (IBD) have an increased risk of bleeding during and after invasive dental interventions, even if the procedures are relatively minor in nature. The intensity and number of bleeds in these patients depend on disease-related factors such as the severity of the bleeding disorder, local and systemic patientrelated factors, as well as intervention-related factors. The patient-related factors include gingival inflammation and blood vessel disease (e.g Raynaud's phenomenon), whilst the intervention-related factors include the number and type of the teeth extracted, or the surface area of the wound following an extraction. ${ }^{1}$

The frequency of bleeding complications after dental treatments in patients with IBDs has been significantly reduced following the introduction of clotting factor 
concentrates, desmopressin (DDAVP) and anti-fibrinolytic agents. ${ }^{2}$ Dental procedures should be scheduled to coincide with administration of FRT to minimize the risks of therapies and to decrease the treatment costs.

The administration of DDAVP 30 to 60 minutes before an invasive procedure is clinically effective in patients with a moderate form of haemophilia $A(H A)$ and for those with type 1 Von Willebrand disease (VWD) and some with type 2 VWD. ${ }^{3}$ Factor Replacement Therapy should be administered 30 minutes to one hour before invasive dental procedures, and the administration repeated 24 hours after the procedure. An additional infusion for patients with HA and VWD must be administered 12 hours after the procedure..$^{3,4}$

The use of clotting factor concentrates comes with the challenge that inhibitors or antibodies are developed towards the replacement factor. 5,6 The use of local haemostatic agents is therefore highly recommended to reduce the risk of postoperative bleeding..$^{7-9}$

Inappropriate dental management of these patients can potentially lead to dangers including airway obstruction as a result of the continued bleeding leading to blood accumulation in the pharynx. Thus it is important for dental practitioners to be knowledgeable about these conditions and to be aware of the guidelines for the management of affected patients. $^{7}$

The aim of this study was to review the records of dental procedures that were performed on patients with IBDs at the WOHC at the CMJAH over a 16-year period.

The objectives of the study were to report on the details of those dental procedures, the occurrence of sustained bleeding and the management thereof, which protocols were observed and how they compared with those published in the literature, and, finally, to make recommendations where necessary. This appears to be the first review of this nature in South Africa.

\section{METHODOLOGY}

Ethical clearance for the study was obtained from the Human Research Ethics Committee of the University of the Witwatersrand, Johannesburg, South Africa (Certificate No. M150425).

The study population comprised all the patients with IBDs referred from the Haemophilia Comprehensive Care Centre (HCCC) at the CMJAH for dental procedures at the Wits Oral Health Centre $(\mathrm{WOHC})$, during the period of January 2000 to December 2015.

The patient data evaluation was retrospective and anonymous and therefore informed consent was not required. Permission to access records at the Haematology unit and the Wits Oral Health Centre was sought and received from the Head of Unit and Head of School.

The IBDs that were included in the study were haemophilia A $(H A)$ and $B(H B)$, Von Willebrand disease (VWD) and other coagulation bleeding diatheses. Patients with inherited platelet disorders, and those with acquired bleeding disorders were excluded from the study as their numbers were generally small in the HCCC and none had undergone dental procedures.

Data were extracted from the HCCC and WOHC patient files and included demographic characteristics, bleeding condition and severity, dental procedure information and replacement therapy, and post-operative bleeding complications. Each eligible patient record was assigned a study number and the data were anonymized. Patients with incomplete file records and missing data relevant to the study were excluded. Data analysis was performed using a statistics programme (STATA). Quantitative data was summarized in tables and figures and described using medians and ranges; counts with percentages.

\section{RESULTS}

Study population demographics

A total of 39 of 106 patients from the HCCC met the study inclusion criteria. Only 21 patient files with the complete data set required for the study were available from the WOHC.

The demographic and baseline characteristics of the study population are depicted in Table 1. The majority of the study population was male (81\%) and black (52\%).

The average age was 26 with a range of 3 to 61 years. The HA patients comprised $48 \%$ of the study population, WWD patients made up 33\%, HB patients 14\%, and only $5 \%$ had factor $\mathrm{V}(\mathrm{FV})$ deficiency.

\begin{tabular}{|c|c|}
\hline Variable & $(\mathrm{N}=21)$ \\
\hline Age, Average (Range) & $26(3-61)$ \\
\hline \multicolumn{2}{|l|}{ Sex, n (\%) } \\
\hline Male & $17(81)$ \\
\hline Female & $4(19)$ \\
\hline \multicolumn{2}{|l|}{ Race, n (\%) } \\
\hline Black & $11(52)$ \\
\hline White & $8(38)$ \\
\hline Indian & $1(5)$ \\
\hline Coloured & $1(5)$ \\
\hline \multicolumn{2}{|l|}{ IBD, n (\%) } \\
\hline Haemophilia A & $10(48)$ \\
\hline Haemophilia B & $3(14)$ \\
\hline Von Willebrand & $7(33)$ \\
\hline Factor $\mathrm{V}$ deficiency & $1(5)$ \\
\hline *IBD: inherited bleeding $d$ & \\
\hline
\end{tabular}

\section{Dental procedures performed on IBD patients}

Figure 1 presents the dental procedures performed during the period of $2000-2015$. There were 33 procedures of which 19 (58\%) were dental extractions.

One patient (3\%) received a scaling and polishing with full mouth debridement (root planing). 


\section{Replacement therapy and anti-fibrinolytics}

Administration of FRT and/or tranexamic acid (TA) in conjunction with the different dental procedures is depicted in Figure 2. Factor Replacement Therapy and TA in a combined administration had been delivered to all the patients who had undergone extractions, in three patients who had received restorative procedures, and in one patient who had undergone extirpation of the pulp and a restorative procedure.

Patients who had undergone scaling and polishing, and one who had undergone fissure sealant placement, did not receive FRT or TA. The administration of FRT was recorded for one subject who had scaling and polishing together with full mouth root planing. Factor Replacement Therapy and TA administration amongst this population was further categorised according to severity (Figure 3). Four subjects who had extractions were of the mild bleeding phenotype, two of moderate intensity, 12 severe, and one case was not specified. TA was administered orally to one subject with unknown severity prior to periodontal probing and plaque control, to one subject with unknown severity prior to scaling and polishing, and to three subjects with moderate severity who had undergone restorative procedures.

\section{The use of local haemostatic agents}

In terms of the type of anaesthesia used, $70 \%$ of the dental procedures were performed under local anaesthesia (LA), while the remainder, all extractions, were undertaken under general anaesthesia (GA).

Table 2: Dental extraction in IBD patients

\begin{tabular}{ll} 
Variable & $\begin{array}{l}\text { Number of teeth or } \\
\text { age in years }\end{array}$ \\
\hline $\begin{array}{l}\text { Total number of extracted teeth } \\
\text { Mean per patient (range) }\end{array}$ & 74 \\
\hline Median & $4(1-14)$ \\
\hline Average age (range) & 3 \\
\hline Teeth extracted under GA & $17(3-60)$ \\
\hline Mean per patient(range) & 61 \\
\hline Median & $6(3-14)$ \\
\hline Average age (range) & 5 \\
\hline Teeth extracted under LA & $9(3-21)$ \\
\hline Mean per patient (range) & 13 \\
\hline Median & $1(1-4)$ \\
Average age (range) & 1 \\
\hline *GA: general anaesthesia; LA: local anaesthesia
\end{tabular}

\section{Dental extractions}

A total of 74 teeth were extracted during the period of 2000 to 2015, as depicted in Table 2. The average age of IBD subjects who underwent extractions was 17 years, and the average number of teeth extracted per subject was four. A total of 61 teeth were extracted under GA and 13 teeth under $L A$. The mean average age of subjects who had extractions under GA was nine years, and 27 years for $L A$. The mean average number of teeth extracted under GA was six, and one under LA.

\section{The use of local haemostatic agents}

The use of local haemostatic agents was seen only in the subjects who had undergone extractions (Figure 4) and was not specified in 58\% of the subjects. The combination of surgicel and suture usage was recorded in $16 \%$ of the subjects, the use of suture alone, in $5 \%$ of the subjects, and the combination of suture, surgicel and TA gauze compression in $5 \%$.

\section{Post-operative bleeding}

Post-extraction bleeding complications were experienced in only three extraction subjects, two of whom were severe HA patients and one a mild WWD patient (Table 3). In one of the subjects with severe HA the bleeding was observed 17 days post-extraction of one tooth. The socket of this patient had been treated at the time of the extraction with a combination of suture and surgicel.

The subsequent management of this subject comprised FRT and TA intravenous administration, while TA was also prescribed orally and as a mouthwash. The use of local haemostatic agents was not recorded in the other two subjects with post-extraction bleeding. The severe HA patient had had extraction of eight teeth under GA and experienced bleeding nine days post-extraction. The patient was managed with FRT and TA was administered both intravenously and orally. The mild VWD subject had bleeding two days post-extraction of six teeth under $\mathrm{GA}$, and was managed by oral TA. The severity of the post-operative bleeding could not be established.

\section{Study limitations}

This was a retrospective study, subject to all the limitations of these investigations, for example inadequate records. Indeed, the lack of complete data meant that a large number of patients were excluded. Nearly a third of the patient population at the HCCC (CMJAH) have medical aid cover and can therefore access dental care privately.

Table 3: Post-extraction bleeding complications

\begin{tabular}{|c|c|c|c|c|}
\hline Pathology & Local haemostatic agent & Dental procedure & Start of bleeding & Treatment \\
\hline Severe HA & Suture and surgicel & $\begin{array}{l}\text { Extraction of } 1 \text { tooth } \\
\text { under LA }\end{array}$ & 17 days post procedure & $\begin{array}{l}\text { FRT and TA IV + TA PO } \\
+ \text { TA mouthwash }\end{array}$ \\
\hline Severe HA & None & $\begin{array}{l}\text { Extraction of } 8 \text { teeth } \\
\text { under GA }\end{array}$ & 9 days post procedure & $\begin{array}{l}\text { FRT and TA IV + TA } \\
\text { PO }\end{array}$ \\
\hline Mild VWD & None & $\begin{array}{l}\text { Extraction of } 6 \text { teeth } \\
\text { under GA }\end{array}$ & 2 days post procedure & TA PO \\
\hline
\end{tabular}




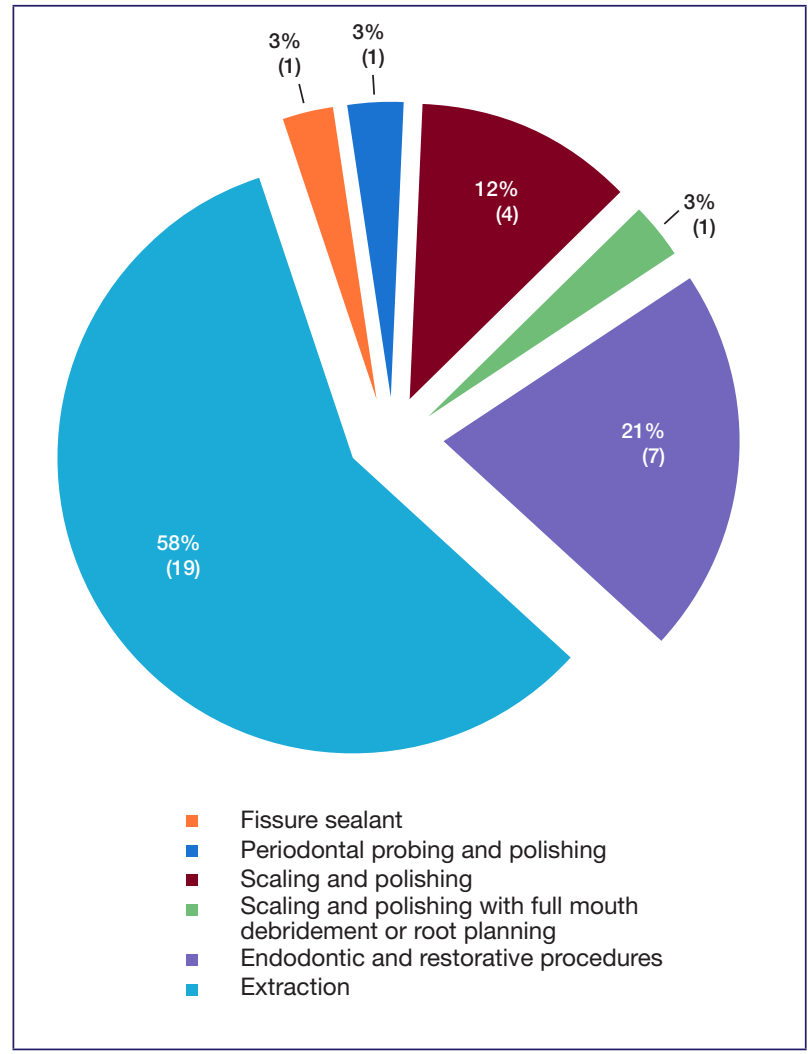

Figure 1. Dental procedures performed during the study period (2000 - 2015).

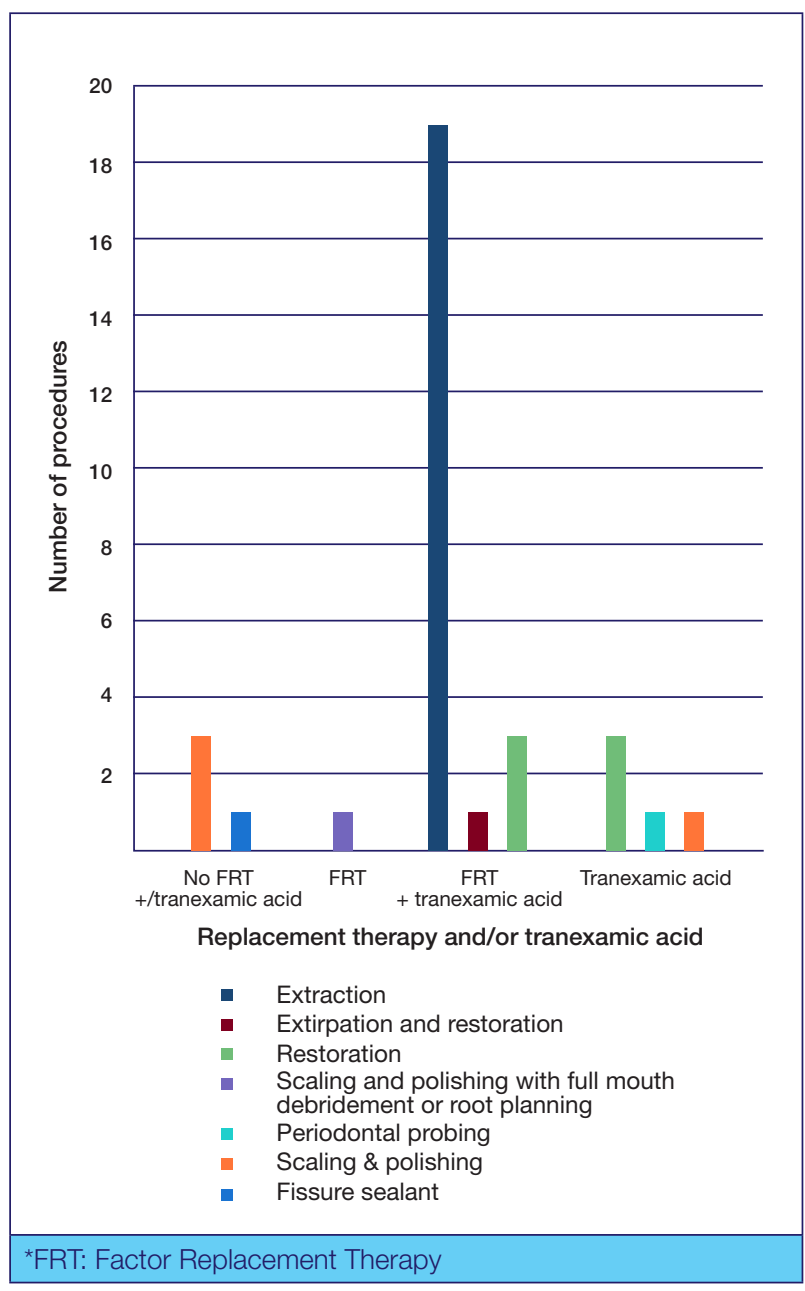

Figure 2. Use of replacement therapy and/or tranexamic acid categorised according to dental procedure.

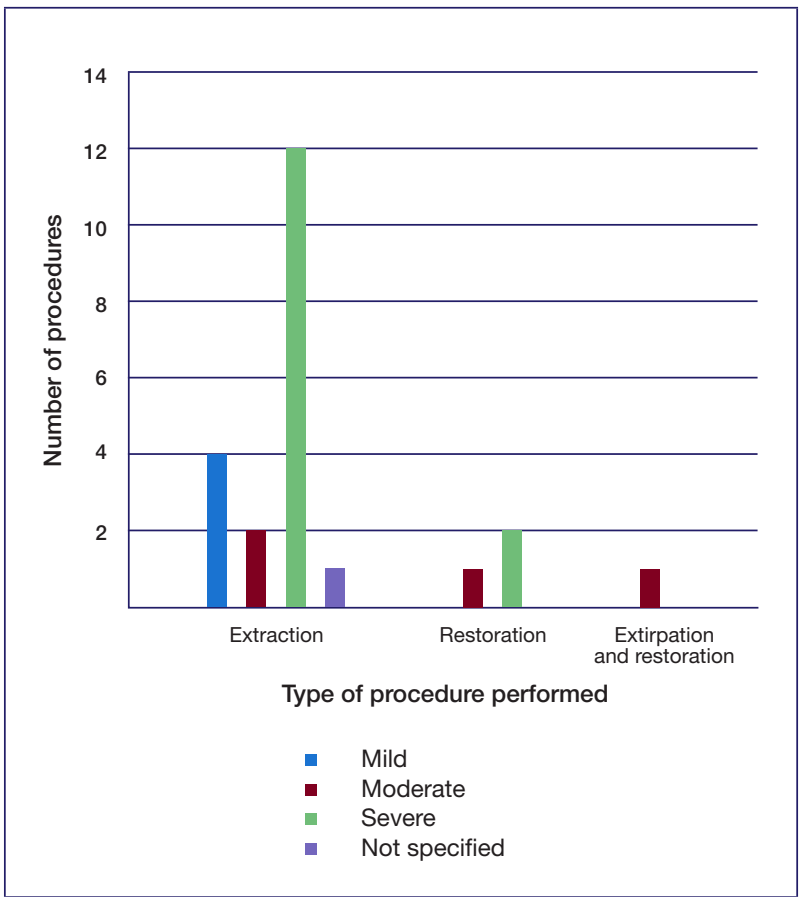

Figure 3. Use of replacement therapy and tranexamic acid categorised according to severity of IBD and dental procedure.

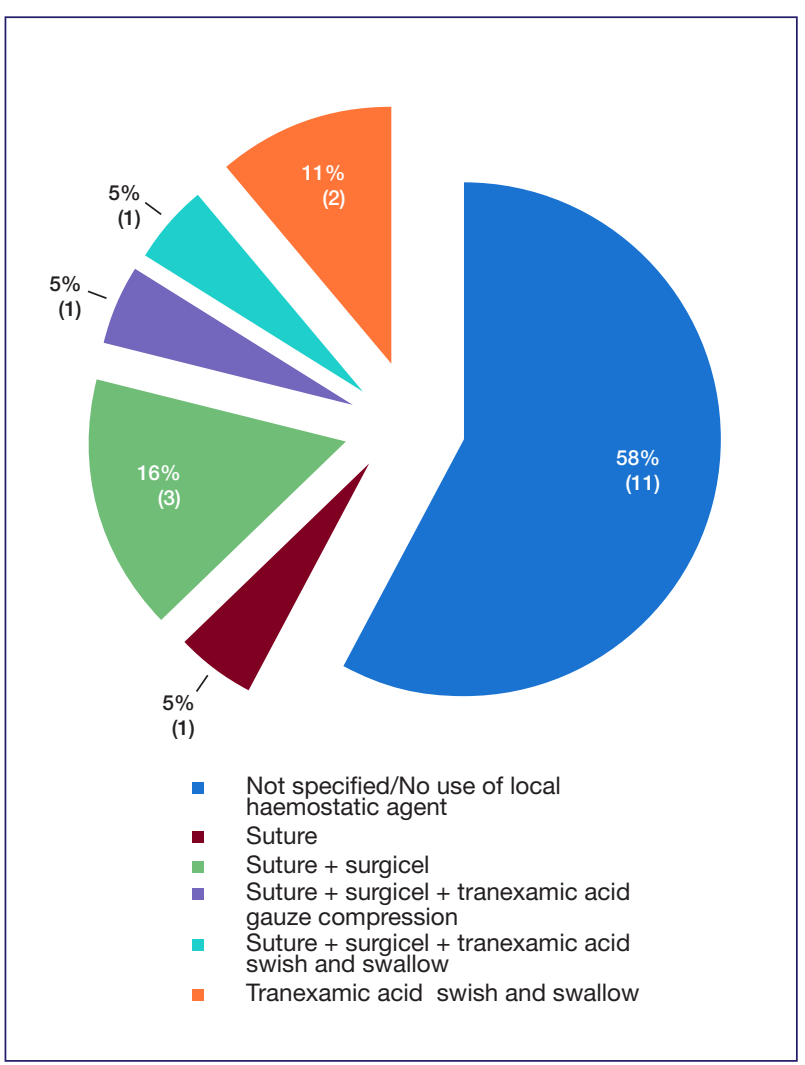

Figure 4. Use of local haemostatic agents in IBD patients. 
Finally, this study raised an important question of poor liaison between the HCCC and WOHC at the $\mathrm{CMJAH}$, evidenced to some extent by the incomplete records.

\section{DISCUSSION AND RECOMMENDATIONS}

A report of the World Health Organization (WHO) states that oral health services in Western industrialized countries comprise preventive and curative services and are available to the general population. The report also states that in many countries in Africa, Asia and Latin-America the majority of services are limited to pain relief or emergency care due to a shortage of oral health personnel. ${ }^{10}$

The public oral health service in South Africa has been described as palliative and demand-driven, since it lacks a structured budget and operational concepts. There is also a critical shortage of oral health professionals in the public sector which leads to the restricted delivery of dental procedures, with the main focus being extractions. ${ }^{11}$

This was attributed to a lack of resources (clinical/technical/ infrastructure) and a high patient load. ${ }^{11}$ This pattern of service delivery observation is reflected in this study, where extraction was the most commonly performed procedure, while preventive services were infrequently rendered.

The average age of subjects who had undergone extractions was 17 years, while the average number of teeth extracted per subject was four. This is a cause for concern as this means that there is premature loss of teeth which impacts on the patient's quality of life.

Dental intervention and promotion of oral health is essential from the early stages of life in order to maintain optimal oral health. ${ }^{12}$ IBD patients are mostly diagnosed in childhood which provides the ideal opportunity for enrolment in a preventive programme so that the need for future invasive procedures can be limited.

Fissure sealant application is recommended as an effective adjunct to other caries preventive procedures. ${ }^{13-15}$ A study conducted in Hammanskraal, an area in the Gauteng Province of South Africa with high natural fluoride levels in the water supply, revealed a $75.2 \%$ reduction in caries levels amongst 15 year-old children who had twice been treated with fissure sealants over a seven year period. ${ }^{13}$

This study set out to examine the management of dental patients who suffered inherited bleeding disorders and to assess the protocols followed at $\mathrm{WOHC}$ and $\mathrm{CMAH}$ in comparison with international standards. The use of replacement therapy, alone and in combination with TA, was observed among the subjects who had undergone extractions, full mouth root planing with scaling and polishing, restorative, and endodontic procedures.

According to the published protocols, extractions and root planing are considered as invasive procedures that are associated with a high risk of bleeding, and therefore require replacement therapy to be administered 30 minutes to one hour before the procedure. This study showed that patients undergoing invasive procedures were managed in accord with the published norms. Restorative and endodontic procedures, however, are associated with a low risk of bleeding, and therefore it is recommended that replacement therapy administration is not required, $, 7,16$ however, in this study, some such patients were in fact also managed with replacement therapy (RT).

No replacement therapy or TA was administered in subjects who had undergone scaling and polishing, and fissure sealants. This practice is in keeping with the published protocols. Periodontal probing and supragingival scaling and polishing are unlikely to cause prolonged bleeding.

Management of patients with poor gingival health requires several visits to prevent excessive bleeding. . $^{7,16}$ It is advised that supragingival scaling along with oral hygiene education and the use of chlorhexidine gluconate mouthwash be initially instituted, so that subgingival scaling can be carried out after the gingival inflammation has subsided. ${ }^{16,17}$ The use of a TA mouthwash after dental scaling in patients with haemophilia has been shown to be as effective in controlling gingival haemorrhage as using FRT. ${ }^{19}$ This implies that FRT can be reserved for more severe bleeding situations. ${ }^{18}$

The use of FRT in conjunction with TA or an anti-fibrinolytic agent, as observed in this study, has been described and is supported by literature. Prior to the introduction of viral inactivated plasma derived factor concentrates, the aim in the management of patients with IBD was to reduce the amount of blood products used wherever it was safe to do so, thereby minimising the possibility of transmitting viral infections.

Pursuant to this objective, clot lysis was impeded in a group undergoing extractions by using replacement therapy in conjunction with epsilon aminocaproic acid (EACA), a plasminogen activation inhibitor. EACA was administered IV prior to and post-extraction followed by oral administration four times daily for 10 days.

This treatment approach is effective in the management of patients with IBDs requiring dental extractions, as the frequency of administration and total quantity of concentrates used could be drastically reduced. ${ }^{18}$ More recently, replacement of recombinant factor has been used to reduce the risk of transmitting blood borne infections. ${ }^{19}$ However it is expensive and may elicit antibodies which destroy the clotting factor. Whilst replacement therapy in combination with anti-fibrinolytic therapy is more effective in reducing blood loss than the use of FRT alone, ${ }^{7,18}$ TA is currently the preferred choice because it is more potent than EACA. ${ }^{19}$

Sixty-one teeth were extracted under GA with the average number of teeth extracted per subject being six, and the average age of subjects at nine years. According to the published protocols GA should be avoided in IBD patients as the procedure can cause a laryngeal haematoma which can lead to upper airway obstruction. . $20^{3}$ The recommendation is that alternatives to GA, such as anxiolytic agents and appropriate sedation, be explored. ${ }^{6}$

LA containing a vasoconstrictor reduces the bleeding during the procedure and also promotes local haemostasis. ${ }^{20}$ However administering the LA may be 
associated with a significant risk of haematoma formation, especially involving the inferior alveolar and posterior superior alveolar nerve blocks, lingual infiltration, and floor-of-mouth injections.

FRT with or without TA is necessary prior to these LA techniques being performed. ${ }^{6}$ The type of LA and the techniques used could not be reported on in this study due to the relevant information not being included in the patients' files.

The high fibrinolytic activity in the oral cavity due to saliva and local tissue plasminogen activator (t-PA) production, qualifies the use of haemostatic agents which inhibit this action and assist in minimizing intra-operative and post-operative bleeding., ${ }^{1,16}$

The agents augment the coagulation cascade, increase the rate of vasoconstriction, seal blood vessels or vascular channels, or promote platelet aggregation. ${ }^{21}$ Their use is therefore very beneficial, and should not be neglected. ${ }^{7-9}$ In this study, local haemostatic agents were recorded in only $42 \%$ of the study population, in whom it was reserved for extractions.

In the current study TA was used post-operatively alone as a swish and swallow in $11 \%$ of the subjects who had extractions, and in conjunction with suture and surgicel in $5 \%$ of those subjects. The literature recommends using TA mouthwash prior to a dental procedure in which bleeding is anticipated, inclusive of scaling and polishing in individuals with poor gingival health. ${ }^{7,19}$

Following the use of TA mouthwash, its concentration in saliva was very high after 30 minutes; above 200 microgams per milliliter, and it remained at a therapeutic level for more than two hours thereafter. ${ }^{22}$ However, the systemic administration of TA resulted in undetectable salivary levels; hence local administration of an anti-fibrinolytic agent is recommended. ${ }^{19,22}$

To make up a 5\% mouthwash solution, a 500mg TA tablet is crushed and dissolved in $10 \mathrm{ml}$ water. TA mouthwash should be swirled in the mouth for 2-3 minutes and either swallowed or expelled. Children should be supervised to ensure that the rinse is expelled to avoid exceeding the recommended dose. This should be done at least 30 minutes before the procedure, and continued six hourly for $7-10$ days.

Management protocols for extraction or surgical sites differ, and include the use of two 500mg ampoules of TA to irrigate the socket or surgical site prior to suturing. ${ }^{9}$ Franchon et al. described a different approach in which the site is cleansed, and a combination of fibrinogen-based biological glue and calcium thrombin is inserted.

This is followed by the insertion of a resorbable gelatin packing, which is then covered with a layer of biological glue. Resorbable sutures are placed and covered with another layer of biological glue. Intermittent TA compressions are applied after the procedure and for the first three days after the procedure, repeated one hourly on the first day, two hourly on the second day and three hourly on the third day. ${ }^{20}$
The recommendation is that local haemostatic agents should be used as described in the literature. A protocol for the management of extraction or surgical sites should be put in place to ensure uniformity in the management of patients.

Even with careful preoperative planning, postoperative bleeding may occasionally be seen. ${ }^{19}$ In the aforementioned study by Franchon et al. post-extraction bleeding was observed at minimum on day one, and at most on day eight in six of the 19 patients that had extractions, despite the extraction site management protocol having been instituted. ${ }^{20}$

In the current study postoperative bleeding was observed in three subjects who had extractions performed. In only one of these subjects was a local haemostatic agent applied, and we may attribute the post-extraction bleeding to factors such as lack of home care or patients not following postoperative instructions. The post-extraction bleeding observed in the other two patients could possibly be attributed to the lack of local haemostatic agents. ${ }^{20}$

Management of post-extraction bleeding includes administration of additional systemic haemostatic therapy, repeated local compression, and the use of a local anaesthetic containing a vasoconstrictor. ${ }^{4,20}$ The protocols for the management of post-operative bleeding correlated with those published.

\section{CONCLUSION}

Successful dental management of patients with IBD must be a joint approach between the dentist and haematologist. ${ }^{7,23}$ Most of the protocols for the dental management of IBD patients observed in this study are similar to those that are published and used internationally. ${ }^{1-9}$ Some of the recommended improvements include the greater use of preventive and supportive dental procedures, local haemostatic agents and the use of LA instead of GA.

A standard protocol for the dental management of IBD patients should be formulated and put in place in $\mathrm{CMJAH}$ $\mathrm{WOHC}$ and at similar facilities to ensure uniformity in the management of IBD patients, and to ensure alignment with internationally accepted norms.

\section{References}

1. van Galen KPM, Engelen ET, Mauser-Bunschoten EP, van Es RJJ, Schutgens REG. Anti-fibrinolytic therapy for preventing oral bleeding in patients with haemophilia or von Willebrand disease undergoing minor oral surgery or dental extractions (Review). CDSR 2015;12:1-33.

2. Franchini M, Rossetti G, Tagliaferri A, Pattacini C, Pozzoli D, Lorenz C, et al. Dental procedures in adult patients with hereditary bleeding disorders: 10 years experience in three Italian Hemophilia Centers. Haemophilia 2005;11:504-9.

3. Piot B, Sigaud-Fiks M, Huet P, Fressinaud E, Trossaërt $\mathrm{M}$, Mercier J, et al. Management of dental extractions in patients with bleeding disorders. Oral Surg Oral Med Oral Pathol Oral Radiol Endod. 2002;93:247-50. 
4. Hewson ID, Daly J, Hallet KB, Liberali SA, Scott CLM, Spaile $G$, et al. Consensus statement by hospital-based dentists providing dental treatment for patients with inherited bleeding disorders. Aust Dent J. 2011;56:221-6.

5. Brewer A, Roebuck E, Donachie M, Hazard A, Gordon K, Fung D, et al. The dental management of adult patients with haemophilia and other congenital bleeding disorders. Haemophilia 2003;9:673-7.

6. Gupta A, Epstein JB, Cabay RJ. Bleeding Disorders of Importance in Dental Care and Related Patient Management. JCDA. 2007;73(1):77-83

7. Anderson JAM, Brewer A, Creagh D, Hook S, Mainwaring J, McKernan A, et al. Guidance on the dental management of patients with haemophilia and congenital bleeding disorders. BDJ. 2013;215:497-504.

8. Givol N, Hirschhorn A, Lubetsky A, Bashari D, Kenet G. Oral surgery-associated postoperative bleeding in haemophilia patients - a tertiary centre's two decade experience. Haemophilia 2015;21:234-40.

9. Federici AB, Sacco R, Stabile F, Carpenedo M, Zingaro E, Mannucci PM. Optimising local therapy during oral surgery in patients with von Willebrand disease: effective results from a retrospective analysis of 63 cases. Haemophilia 2000;6:71-7.

10. www.who.int/oral_health/action accessed on the 24 February 2017.

11. Mickenautsch S, Van't Hof MA, Frencken JE. Oral health service systems in Gauteng Province, South Africa. East Afr Med J. 2007;84(4):178-82.

12. Kabil N, El Alfy M, Metwalli N. Evaluation of the oral health situation of a group of Egyptian haemophilic children and their re-evaluation following an oral hygiene and diet education programme. Haemophilia 2007;13:287-92.

13. van Wyk PJ, Kroon J, White JG. Evaluation of a fissure sealant program as part of community-based teaching and training. JDE. 2004;68(1):50-4.

14. Beauchamp J, Caufield PW, Crall JJ, Donly D, Feigal R, Gooch B, et al. Evidence-based clinical recommendations for the use of pit-and-fissure sealants. A report of the American Dental Association Council on Scientific Affairs. JADA. 2008;139:257-68.
15. Wright JT, Crall JJ, Fontana M, Gillette EJ, Nový $\mathrm{BB}$, Dhar $\mathrm{V}$ et al. Evidence-based clinical practice guideline for the use of pit-and-fissure sealants. A Report of the American Dental Association and the American Academy of Pediatric Dentistry. JADA. 2016;147:672-82.

16. http://www.ahcdo.org.au/sitebuilder/publications/ knowledge/asset/files/16/dentalguidelinesforwebsitedec10.pdf A consensus statement on the dental treatment of patients with inherited bleeding disorders. Australian Haemophilia Centre Directors' Organisation July 2010. Accessed on the 22 April 2014.

17. Brewer A, Correa ME. Guidelines for dental treatment of patients with inherited bleeding disorders. Treatment of Hemophilia 2006;40:1-9.

18. Walsh PN, Rizza CR, Matthews JM, Eipe J, Kernoff PBA, Coles MD, et al. Epsilon-Aminocaproic acid therapy for dental extractions in haemophilia and Christmas disease: a double blind controlled trial. $\mathrm{Br} \mathrm{J}$ Haematol. 1971;463(20):463-75.

19. Lee APH, Boyle CA, Savidge GF, Fiske J. Effectiveness in controlling haemorrhage after dental scaling in people with haemophilia by using tranexamic acid mouthwash. BDJ. 2005;198(1):33-8.

20. Franchon X, Pommereuil M, Berthier A, Lejeune S, Hourdin-Eude S, Quéro J, et al. Management options for dental extraction in hemophiliacs: A study of 55 extractions (2000-2002). Oral Surg Oral Med Oral Pathol Oral Radiol Endod. 2005;99:270-5.

21. Ogle OE, Swantek J, Kamoh A. Hemostatic agents. Dent Clin N Am. 2011;55:433-9.

22. Sindet-Pedersen S. Distribution of tranexamic acid to plasma and saliva after oral administration and mouth rinsing: a pharmacokinetic study. The Journal of Clinical Pharmacology 1987;27(12):1005-8.

23. Stubbs M, Lloyd J. A protocol for the dental management of von Willebrand's disease, haemophilia A and haemophilia B. Aus Dent J. 2001;46(1):37-40.

\section{ERRATUM}

Sadly, the Journal has incorrectly recorded the name of one of our authors and an apology is sincerely extended. The article appeared in the June 2018 issue under the title: "Early childhood caries experience of children accessing selected immunization facilities in Johannesburg."

Reference 2018;73(5):330.

WG Evans: Managing editor, Email: bill.evans@wits.ac.za
The name of the first author was recorded as NP Molete and a first name of Primrose appeared. The name correctly should have been MM Molete with first names Mpho Matlakale.

Figure 1 showed an arrow slanted at an angle... this should have been horizontal, not angled.

The Journal regrets these errors and thanks Dr Molete for drawing attention to the glitches. 